\title{
Evaluation of White Matter Tracts Fractional Anisotropy Using Tract-Based Spatial Statistics and Its correlation with Amyotrophic Lateral Sclerosis Functional Rating Scale Score in Patients with Motor Neuron Disease
}

\author{
Amutha Bharathi Mohan ${ }^{1}$ Subathra Adithan ${ }^{1, \odot}$ \\ Donna Mathews 2,3 \\ ${ }^{1}$ Department of Radiodiagnosis, Jawaharlal Institute of \\ Postgraduate Medical Education and Research (JIPMER), \\ Puducherry, India \\ 2Department of Neurology, Jawaharlal Institute of Postgraduate \\ Medical Education and Research (JIPMER), Puducherry, India \\ ${ }^{3}$ Department of Neurology, Christian Medical College (CMC), \\ Vellore, Tamil Nadu, India
}

Sunil Narayan² Nagarajan Krishnan ${ }^{1}$

\begin{abstract}
Address for correspondence Subathra Adithan, MD, Department of Radiodiagnosis, Jawaharlal Institute of Postgraduate Medical Education and Research (JIPMER), Puducherry 605006, India (e-mail: subathra26@gmail.com).
\end{abstract}

\begin{abstract}
Keywords

- motor neuron disease

- TBSS

- amyotrophic lateral sclerosis

- fractional anisotropy

Background Motor neuron diseases cause progressive degeneration of upper and lower motor neurons. No Indian studies are available on diffusion tensor imaging (DTI) findings in these patients.

Aims This study was done to identify white matter tracts that have reduced fractional anisotropy (FA) in motor neuron disease (MND) patients using tract-based spatial statistics and to correlate FA values with Amyotrophic Lateral Sclerosis Functional Rating Scale (ALSFRS-R) score.

Settings and Design A case-control study in a tertiary care hospital.

Materials and Methods We did DTI sequence (20 gradient directions, $b$-value 1,000) in 15 MND patients ( 10 men and 5 women; mean age: $46.5 \pm 16.5$ years; 11 amyotrophic lateral sclerosis [ALS], 2 monomelic amyotrophy, 1 progressive muscular atrophy, and 1 bulbar ALS) and 15 age- and sex-matched controls. The data set from each subject was postprocessed using FSL downloaded from the FMRIB Software Library, Oxford, United Kingdom (http://www.fmrib.ox.ac.uk/fsl).

Statistical Analysis The statistical permutation tool "randomize" with 5,000 permutations was used to identify voxels that were different between the patient data set and the control data set. Mean FA values of these voxels were obtained separately for each tract as per "JHU white-matter tractography atlas." SPSS was used to look to correlate tract-wise mean FA value with ALSFRS-R score.
\end{abstract}

published online July 28,2021
DOI https://doi.org/

$10.1055 / \mathrm{s}-0041-1734337$ ISSN 0971-3026
(C) 2021. Indian Radiological Association.

This is an open access article published by Thieme under the terms of the Creative Commons Attribution-NonDerivative-NonCommercial-License, permitting copying and reproduction so long as the original work is given appropriate credit. Contents may not be used for commercial purposes, or adapted, remixed, transformed or built upon. (https://creativecommons.org/licenses/by-nc-nd/4.0/). Thieme Medical and Scientific Publishers Private Ltd. A-12, Second Floor, Sector -2, NOIDA -201301, India 
Results We found clusters of reduced FA values in multiple tracts in the brain of patients with MND. Receiver operating characteristic curves plotted for individual tracts, showed that bilateral corticospinal tract, bilateral anterior thalamic radiation, bilateral uncinate fasciculus, and right superior longitudinal fasciculus were the best discriminators (area under the curve $>0.8, p<0.01$ ). FA values did not correlate with ALFRS-R severity score.

Conclusion In MND patients, not only the motor tracts, but several nonmotor association tracts are additionally affected, reflecting nonmotor pathological processes in ALS.

\section{Introduction}

Motor neuron disease (MND) is a disease involving progressive degeneration of upper (UMN) and lower motor neurons (LMN). It is incurable, though the rate of progression may vary. Amyotrophic lateral sclerosis (ALS) is the most common type of MND. Its incidence varies between 1 to 2.6 per 100,000 annually, and the prevalence is 6 per $100,000^{1}$; the number of cases is projected to increase by $69 \%$ over the next 25 years. ${ }^{2}$ There are other types of MND such as progressive bulbar palsy, progressive muscular atrophy, primary lateral sclerosis, spinal muscular atrophy, pseudobulbar palsy, and monomelic amyotrophy (MMA). MMA is a rare cervical myelopathy that manifests as a self-limited, asymmetrical, slowly progressive atrophy with minimal weakness of the forearms and hands, predominantly in young males.

Many times, patients with MND present with predominantly LMN dysfunction, and it is not possible to predict whether they will progress to UMN dysfunction. MND has a variable prognosis. ALS has a very aggressive course and grave prognosis, whereas MMA has a more protracted course often with nonprogressive stabilization of deficits. Several studies have shown decreased fractional anisotropy (FA) in white matter tracts of patients with $\mathrm{MND}^{3-5}$ but measuring FA in MND patients has not found its place in routine clinical practice as of now.

We found no published studies on imaging findings of MND in the Indian population. Anatomical signatures of different types of MND may be different. Studies have found specific patterns of white matter involvement associated with certain genetic mutations such as diffuse white matter abnormalities involving the corticospinal tract, whole corpus callosum, and superior longitudinal fasciculus in C9orf72 repeat expansion carriers (the most common genetic mutation in ALS patients). ${ }^{6}$

Data on the patterns of involvement of white matter tracts in Indian patients are not present. Therefore, the present study was done to identify the white matter tracts that are affected by MND, using FA as a tool, through an automated process-tract-based spatial statistics (TBSS). We also tried to correlate the degree of FA changes in the white matter tracts with loss of functionality as revealed by the Amyotrophic Lateral Sclerosis Functional Rating Scale (ALSFRS-R) score.

\section{Subjects and Methods}

This case-control study was conducted from June 2014 to July 2016 in the departments of radiodiagnosis and neurology in a tertiary care hospital in South India on 15 patients diagnosed to have MND and 15 age- and sex-matched controls.

\section{Study Procedure}

This study was approved by the institute ethics board. Written informed consent was obtained from all patients and controls. Relevant investigations, including electrophysiological studies, were done to rule out secondary causes of motor system diseases. A neurologist with more than 30 years of experience diagnosed the patients with MND. Disease severity was scored as per ALSFRS-R scale (greater the disease severity, lesser the score). ${ }^{7}$ Disease duration was recorded in months. Controls were matched for age and sex to patients. Controls were examined clinically and by magnetic resonance imaging (MRI) and were found to be normal. Diffusion tensor imaging (DTI) data sets (20 gradient directions, number of acquisitions $4, b$-value 1,000, TR: 3,700 ms, TE: $88 \mathrm{~ms}$, matrix: $128 \times 128$ ) were acquired with a $1.5 \mathrm{~T}$ (Siemens, Erlangen, Germany) machine in both patients and controls.

\section{FA Estimation Methods}

TBSS was used to compare the FA index of white matter tracts between patients and controls.

All data sets are analyzed using FSL version 5.0.8, part of the Functional Magnetic Resonance of the Brain (FMRIB) Software Library, Oxford, United Kingdom (http://www. fmrib.ox.ac.uk/fsl).

\section{Tract-Based Spatial Statistics methodology}

The image processing and analysis workflow consisted of four steps:

(1) Image preprocessing to setup DTI data for TBSS; (2) TBSS; (3) voxel-wise statistical analysis; and (4) review of output. 
Step 1: Preprocessing for Setting up Data for TBSS

Digital Imaging and Communications in Medicine images were converted to Neuroimaging Informatics Technology Initiative (NifTI) format using the dcm2nii conversion tool from the MRIcron package that can be downloaded from https://people.cas.sc.edu/rorden/mricron/dcm2nii.html.

From dcm2nii, three files were obtained-diffusion tensor data file in the Nifti format, Bvec file that contains data on gradient directions, and Bval file that provides information on the magnitude of gradients. Further steps were done to prepare data for TBSS as described below in each subject's data file:

1. Eddy current distortion correction.

2. Binary brain mask extraction using BET tool to strip the skull of bone and include only brain parenchyma.

3. Fitting diffusion tensor to each voxel to get FA index using FMRIB's Diffusion Toolbox 3.0, DTIFIT.

\section{Step 2: TBSS to Obtain the Skeletonized Data File}

After checking the quality of output files for each subject, all the files were put in a single directory and scripts were run on them to align the data files to a standard template $(1 \times 1$ $\times 1 \mathrm{~mm}$ T1-weighted image; Montreal Neurological Institute 152 Template).

FA maps of all subjects were reduced to a skeleton. This form reduced partial volume effects and removed peripheral white matter structures with high variability. The threshold of 0.2 was taken to include only significant tracts in the mean FA skeleton. It also created a mean FA skeleton mask.

\section{Step 3: Statistical Analysis of Skeletonized Data}

"Randomize tool" with age as an explanatory variable was used from FSL (Randomize FSL; FMRIB Software Library) with Threshold-Free Cluster Enhancement to detect clusters that were different between patient group and control group at a $p$-value of 0.05 , after family-wise error corrections for multiple comparisons. The number of permutations was set to 5,000 .

\section{Step 4: Review of Output}

FSLeyes was used to view the results. The clusters were annotated using the Johns Hopkins University white-matter tractography atlas. Mean FA values of individual tracts were obtained using the "cluster" command.

The same procedure (step 2 to step 4) was repeated to compare mean diffusivity (MD) and radial diffusivity (RD) between patients and controls.

\section{Statistical Analysis}

FA values were summarized in terms of mean \pm standard deviation for different white matter tracts in patients (for each type of MND) and controls. The data was entered in Redcap (version 6.11.1). SPSS software (version 20) was used for analyzing data. Receiver operating characteristic (ROC) curve was plotted separately for each white matter tract; the area under the curve (AUC) was obtained for the same. FA values in individual white matter tracts were correlated with disease severity (ALSFRS-R score) and disease duration using Spearman's rho correlation in patients. Bonferroni correction was used for multiple comparisons, and $p<0.01$ was taken as significant.

\section{Results}

\section{Patient Characteristics and Conventional MRI Findings}

Fifteen patients (11 ALS, 1 MMA, 1 progressive muscular atrophy, and 1 bulbar ALS; 10 men and 5 women; mean age 46.5 \pm 16.5 years) and 15 controls ( 12 men and 3 women; mean age $44.6 \pm 9.8$ years) participated in the study. The average duration of disease in patients was $4.4 \pm 10$ years. Patients and control groups showed no significant differences regarding age $(p=0.70)$ and sex $(p=0.42)$. The mean ALSFRS-R score was $38.53 \pm 5.63$ (range: $25-45$ ).

$\mathrm{T} 1, \mathrm{~T} 2$, and fluid-attenuated inversion recovery sequences of the brain were unremarkable in 7 patients, nonspecific white matter hyperintensities were seen in 7 patients, and 1 patient had mild cerebral and cerebellar atrophy. None of the patients showed hyperintensity along the corticospinal tract or selective motor cortex atrophy.

Clusters with reduced FA value $(p<0.05)$ were found in several white matter tracts in MND patients (-Fig. 1), namely,

(1) bilateral anterior thalamic radiation,

(2) bilateral corticospinal tracts,

(3) bilateral cingulum (cingulate gyrus, hippocampus),

(4) bilateral inferior fronto-occipital fasciculus,

(5) bilateral inferior longitudinal fasciculus,

(6) bilateral superior longitudinal fasciculus,

(7) bilateral superior temporal longitudinal fasciculus,

(8) bilateral uncinate fasciculus,

(9) forceps major, and

(10) forceps minor.

Results of ROC curves plotted using mean FA values extracted from each of these tracts for ALS patients is shown in - Table 1. The tracts that show the best discriminatory value (AUC > 0.8) between ALS patients and controls are left corticospinal tract, right corticospinal tract, right anterior thalamic radiation, right uncinate fasciculus, right superior longitudinal fasciculus, and left uncinate fasciculus.

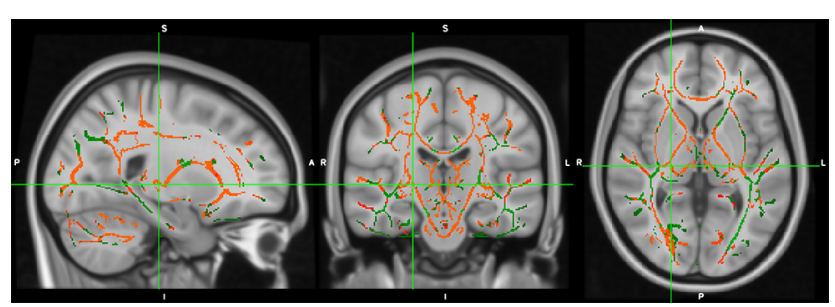

Fig. 1 Clusters showing reduced fractional anisotropy at $p<$ 0.05 overlaid in orange over a standard Montreal Neurological Institute (MNI) 152_T1_1 $1 \mathrm{~mm}$ image. Mean fractional anisotropy (FA) skeleton is overlaid in green. 
Table 1 ROC analysis in TBSS method showing AUC of various white matter regions

\begin{tabular}{|c|c|c|}
\hline White matter tract & $\begin{array}{l}\text { Area under the } \\
\text { curve }(95 \% \mathrm{CI})\end{array}$ & $p$-Value \\
\hline Left corticospinal tract & $0.897(0.778-1.000)$ & 0.001 \\
\hline $\begin{array}{l}\text { Right anterior thalamic } \\
\text { radiation }\end{array}$ & $0.897(0.779-1.000)$ & 0.001 \\
\hline Right uncinate fasciculus & $0.867(0.718-1.000)$ & 0.002 \\
\hline Right corticospinal tract & $0.855(0.707-1.000)$ & 0.002 \\
\hline $\begin{array}{l}\text { Right superior longitudinal } \\
\text { fasciculus }\end{array}$ & $0.818(0.654-0.983)$ & 0.006 \\
\hline Left uncinate fasciculus & $0.812(0.643-0.981)$ & 0.008 \\
\hline $\begin{array}{l}\text { Left anterior thalamic } \\
\text { radiation }\end{array}$ & $0.794(0.620-0.967)$ & 0.012 \\
\hline Forceps major & $0.733(0.533-0.934)$ & 0.046 \\
\hline $\begin{array}{l}\text { Right superior temporal } \\
\text { longitudinal fasciculus }\end{array}$ & $0.727(0.530-0.924)$ & 0.052 \\
\hline Left cingulate gyrus & $0.709(0.510-0.908)$ & 0.073 \\
\hline $\begin{array}{l}\text { Right inferior fronto-occipital } \\
\text { fasciculus }\end{array}$ & $0.709(0.508-0.910)$ & 0.073 \\
\hline Forceps minor & $0.685(0.477-892)$ & 0.113 \\
\hline $\begin{array}{l}\text { Left superior longitudinal } \\
\text { fasciculus }\end{array}$ & $0.679(0.464-0.893)$ & 0.126 \\
\hline Left cingulate hippocampus & $0.667(0.444-0.889)$ & 0.154 \\
\hline Right cingulate gyrus & $0.671(0.476-0.866)$ & 0.11 \\
\hline $\begin{array}{l}\text { Left inferior fronto-occipital } \\
\text { fasciculus }\end{array}$ & $0.630(0.413-0.847)$ & 0.264 \\
\hline $\begin{array}{l}\text { Right inferior longitudinal } \\
\text { fasciculus }\end{array}$ & $0.600(0.378-0.822)$ & 0.392 \\
\hline $\begin{array}{l}\text { Left superior temporal longi- } \\
\text { tudinal fasciculus }\end{array}$ & $0.582(0.347-0.817)$ & 0.484 \\
\hline Right cingulate hippocampus & $0.552(0.316-0.787)$ & 0.659 \\
\hline $\begin{array}{l}\text { Left inferior longitudinal } \\
\text { fasciculus }\end{array}$ & $0.533(0.302-0.764)$ & 0.775 \\
\hline
\end{tabular}

Abbreviations: AUC, area under the curve; $\mathrm{Cl}$, confidence interval; $\mathrm{ROC}$ receiver operating characteristic; TBSS, tract-based spatial statistics.
Cutoff values to discriminate patients and controls for significant tracts (at $p<0.01$ ), with sensitivity and specificity are given below:

1. left corticospinal tract: 0.117 (sensitivity $93.3 \%$, specificity $80 \%$ ); 0.108 (sensitivity 53.3\%, specificity 99.94\%).

2. right corticospinal tract: 0.115 (sensitivity $80 \%$, specificity 73.3\%); 0.112 (sensitivity 73.3\%, specificity 99.94\%).

3. left anterior thalamic radiation: 0.086 (sensitivity $66.7 \%$, specificity 66.7\%); 0.084 (sensitivity 60\%, specificity $86.7 \%)$.

4. right anterior thalamic radiation: 0.090 (sensitivity $100 \%$, specificity 60\%); 0.085 (sensitivity 60\%, specificity 100\%).

5. left uncinate fasciculus 0.109 (sensitivity $93.3 \%$, specificity 56.7\%); 0.101 (sensitivity 46.7\%, specificity 99.94\%).

6. right uncinate fasciculus: 0.087 (sensitivity 86.7\%, specificity66.7\%);0.082(sensitivity60\%,specificity99.94\%).

7. right superior longitudinal fasciculus: 0.073 (sensitivity $86.7 \%$, specificity $73.3 \%$ ); 0.0689 (sensitivity 60\% and specificity $99.94 \%)$.

- Table 2 shows the mean FA value in these tracts in 11 ALS patients, 2 MMA patients, 1 progressive muscular atrophy patient, and 1 bulbar ALS patient.

When MD and RD were compared between patients and controls, we found clusters with reduced MD ( - Fig. 2) and reduced $\mathrm{RD}(\boldsymbol{-}$ Fig. $\mathbf{3})$ at $p<0.05$. Clusters, though fewer in number, belonged to the same tracts that showed reduced FA, except that there were no clusters from right uncinate fasciculus that showed reduced RD. We did not find any clusters that showed increased MD or RD.

Mean MD and mean RD values of individual extracted tracts, when plotted with ROC curves, did not discriminate between patients and controls at a significant level.

There was no significant correlation between FA values and ALFRS-R severity scores and disease duration (Spearman's correlation). - Fig. 4 shows scatter plots for FA values against disease severity.

Table 2 Mean FA values in tracts that showed a significant difference between ALS patients and controls at a p-value $<0.01$ for various subtypes of MND

\begin{tabular}{|l|l|l|l|l|l|}
\hline $\begin{array}{l}\text { White matter } \\
\text { tract }\end{array}$ & $\begin{array}{l}\text { Mean FA in 11 } \\
\text { ALS patients }\end{array}$ & $\begin{array}{l}\text { Mean FA in 2 } \\
\text { MMA patients }\end{array}$ & $\begin{array}{l}\text { Mean FA in 1 PMA } \\
\text { patient }\end{array}$ & $\begin{array}{l}\text { Mean FA in 1 bulbar } \\
\text { ALS patient }\end{array}$ & Mean FA in controls \\
\hline $\begin{array}{l}\text { Left corticospinal } \\
\text { tract }\end{array}$ & $0.108 \pm 0.006$ & $0.104 \pm 0.005$ & 0.115804 & 0.106754 & $0.118 \pm 0.005$ \\
\hline $\begin{array}{l}\text { Right corticospinal } \\
\text { tract }\end{array}$ & $0.108 \pm 0.007$ & $0.106 \pm 0.007$ & 0.112015 & 0.106486 & $0.118 \pm 0.005$ \\
\hline $\begin{array}{l}\text { Left anterior tha- } \\
\text { lamic radiation }\end{array}$ & $0.082 \pm 0.004$ & $0.084 \pm 0.004$ & 0.089109 & 0.083989 & $0.088 \pm 0.004$ \\
\hline $\begin{array}{l}\text { Right anterior tha- } \\
\text { lamic radiation }\end{array}$ & $0.085 \pm 0.003$ & $0.086 \pm 0.003$ & 0.090424 & 0.08241 & $0.091 \pm 0.004$ \\
\hline $\begin{array}{l}\text { Left uncinate } \\
\text { fasciculus }\end{array}$ & $0.108 \pm 0.006$ & $0.096 \pm 0.012$ & 0.109666 & 0.099671 & $0.108 \pm 0.006$ \\
\hline $\begin{array}{l}\text { Right uncinate } \\
\text { fasciculus }\end{array}$ & $0.081 \pm 0.004$ & $0.077 \pm 0.012$ & 0.090473 & 0.073801 & $0.088 \pm 0.004$ \\
\hline $\begin{array}{l}\text { Right superior lon- } \\
\text { gitudinal fasciculus }\end{array}$ & $0.069 \pm 0.004$ & $0.067 \pm 0.005$ & 0.065878 & 0.066843 & $0.074 \pm 0.005$ \\
\hline
\end{tabular}

Abbreviations: ALS, amyotrophic lateral sclerosis; FA, fractional anisotropy; MMA, monomelic amyotrophy; MND, motor neuron disease; PMA, progressive muscular atrophy. 


\section{Discussion}

Corticospinal tract degeneration in MND modifies diffusion characteristics measured by DTI, resulting in changes in FA. TBSS was developed as an unbiased automated technique in which whole brain-based voxel-wise comparison can be made between groups.

We found that there were voxel clusters that had significantly different FA values in the patient group compared with the control group in the projection fibers (corticospinal tract, anterior thalamic radiation), association fibers (uncinate fasciculus, superior and inferior longitudinal fasciculus, inferior

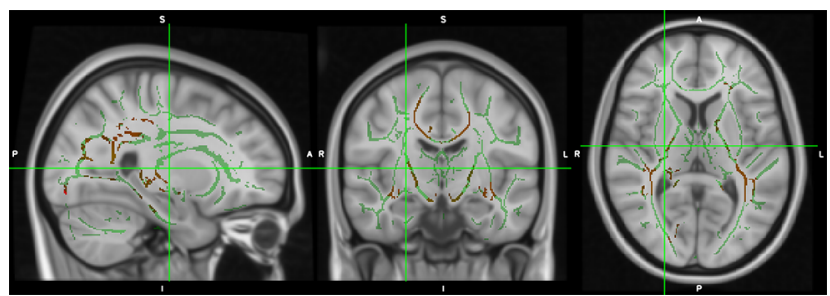

Fig. 2 Clusters showing reduced mean diffusivity (MD) at $p<$ 0.05 overlaid in brown over a standard Montreal Neurological Institute (MNI) 152_T1_1 mm image. Mean MD skeleton is overlaid in green.

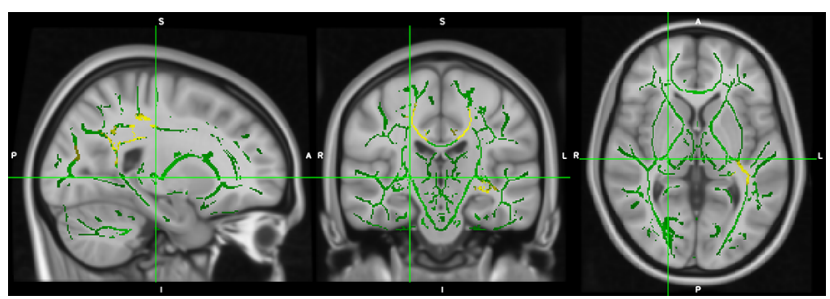

Fig. 3 Clusters showing reduced radial diffusivity (RD) at a $p<$ 0.05 overlaid in yellow over a standard Montreal Neurological Institute (MNI) 152_T1_1 mm image. Mean RD skeleton is overlaid in green. fronto-occipital fasciculus), commissural fibers (forceps major, forceps minor), and limbic system fibers (cingulum).

Similar studies have been done before in the Western population. ${ }^{6}$ Prudlo et al showed widespread white matter changes among all-fiber groups of the brain: projection fibers, commissural fibers, association fibers, and limbic system fibers. ${ }^{8}$ Studies have shown reduced FA values not only in corticospinal tracts but also in several extra-motor regions. ${ }^{9}$

Since the corticospinal and corticobulbar tracts are major efferent projection fibers that connect the motor cortex to the spinal cord and brainstem, reductions in FA value in them may be expected in MNDs. ${ }^{10,11}$ Reduced FA values in the corticospinal tract have been reported in other studies. ${ }^{12-15}$ Further, Wang and Melhem reported that the FA value in the posterior limb of the internal capsule is an optimal site for measurement in patients with MND. ${ }^{15}$

In an Italian study, a reduction in FA values in the anterior thalamic radiation, in addition to the involvement of the corticospinal tract was reported in ALS patients. ${ }^{16}$ They also described increased MD and RD in these tracts. MD is a measure of the average molecular motion independent of any tissue directionality, an overall magnitude of water diffusion. Higher MD indicates an increased volume of extracellular space or decreased barriers to diffusion; it indicates lesser microstructural organization. In our study, we found clusters that showed reduced MD and RD. This paradoxical reduced MD maybe because of partial volume mixing of multiple fiber tracts that cross or touch.

Cingulate gyrus contains small and large pyramidal cells, that project to the motor cortex and spinal cord. It interconnects portions of the frontal, parietal, and temporal lobes. ${ }^{17} \mathrm{Li}$ et al did a systematic review of eight studies and found FA reductions in bilateral frontal white matter/cingulate gyrus and posterior limb of the internal capsule. ${ }^{18}$ They suggested that FA reduction in the frontal white matter and cingulate gyrus may be a unique biomarker of ALS. In our study, we

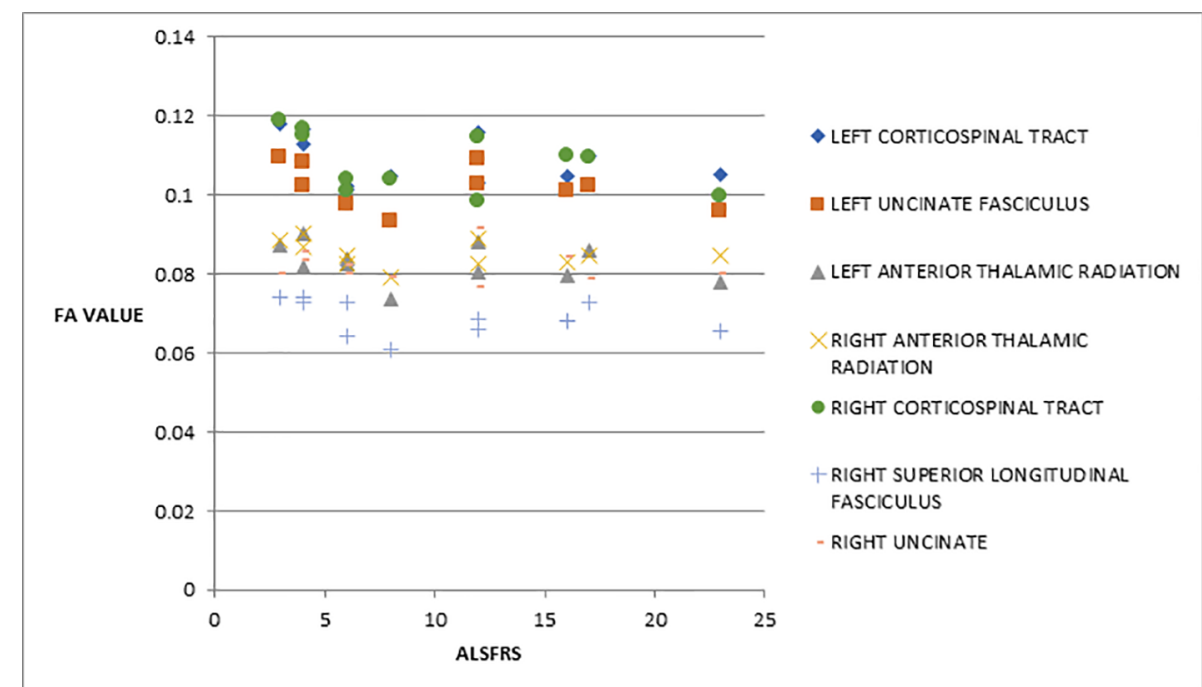

Fig. 4 Scatter plot showing the relationship between fractional anisotropy (FA) value and Amyotrophic Lateral Sclerosis Functional Rating Scale (ALSFRS-R) severity score. 
found clusters with reduced FA values, but they did not reach significance at the tract level.

The uncinate fasciculus hooks around the lateral fissure to connect the orbital and inferior frontal gyri of the frontal lobe to the anterior temporal lobe. Trojsi et al found decreased FA and increased MD and RD in white matter underneath pre- and postcentral gyri, including the bilateral superior and inferior longitudinal and fronto-occipital fasciculi, right uncinate fasciculus. ${ }^{19}$ They also showed changes in several extra-motor frontotemporal regions such as premotor, prefrontal, anterior cingulate, orbitofrontal, parahippocampal cortices, and insular gyri. These changes resemble the cognitive and neuropsychiatric changes typical of frontotemporal degeneration. Kasper et al showed decreased FA values in the inferior fronto-occipital fasciculus, the superior and inferior longitudinal fasciculus, the uncinate fasciculus in addition to corticospinal tract and body of the corpus callosum on comparison to controls. ${ }^{20}$ Their study suggested that the involvement of extra-motor tracts underlies the impairment of cognition in nondemented ALS patients. The frontotemporal dementia (FTD)-ALS phenotype is characterized by marked behavioral abnormalities. There have been reports that uncinate fasciculus involvement may be associated with behavioral symptoms in patients with ALS. ${ }^{21}$ In our study population, the uncinate fasciculus was significantly affected, but we did not find behavioral symptoms in any patients. However, we did not follow-up on these patients. Follow-up of the patients with uncinate fascicular involvement for development of behavioral symptoms of FTD will provide valuable information on the predictive role of this MRI finding. In another study, corpus callosum hippocampal formations, cingulum, and frontal white matter showed DTI changes, which reflected cognitive impairment in ALS patients. ${ }^{22}$

There was no correlation between FA value and disease severity in this study. A longitudinally conducted DTI study by Keil et al showed a positive correlation between FA value and disease severity as per the ALSFRS score in the corticospinal tract and cerebellum. ${ }^{22}$ They have, however, cautioned that ALSFRS score is a composite score of UMN and LMN fibers while FA of corticospinal tract measures predominantly UMN tract degeneration. We did not find a correlation between FA values and disease duration. Inconsistent results have been reported by other studies with regard to FA value and disease duration. ${ }^{6}$

DTI scalar indices are also influenced by acquisition parameters. An increase in the $b$-value of the dephasing and rephasing gradients or an increase in the number of gradient directions is associated with a decrease in FA values. A smaller voxel size is associated with increased FA value. ${ }^{23}$ So FA values can vary with the MRI laboratories/institutional protocols. Recently, an attempt to harmonize acquisition and processing of DTI data that was acquired in four centers has been described by Kalra et al. ${ }^{24}$ In addition, TBSS involves steps such as skeletonization and projection into a normalized template, of white matter with high FA. This misses a portion of the white matter and also misses fibers that cross and have reduced FA due to the crossing. ${ }^{25}$ Further, all voxels corresponding to a tract defined by an atlas is used in TBSS, and reduced FA values in a certain proportion of voxels can bring down the mean FA value of the tract.

Prokscha et al suggest that a method combining atlas-based ROI with TBSS holds promise in developing FA as a marker of cerebral degeneration in ALS. ${ }^{11}$

Studies done in Europe have described C9orf77 as the most common genetic mutation in ALS patients and have reported that these patients have diffuse white matter abnormalities, while patients who were C9orf72 negative showed limited extra-motor pathology. ${ }^{6,26,27}$ The mutation frequency varies with ethnicity; a recent study by Shamim et al has described the genetic profile in 593 Indian patients. ${ }^{28}$ In this context, it is pertinent to describe the pattern of white matter abnormalities found in the Indian population and build data on the phenotypic profile of white matter changes in the Indian patients.

It will be helpful to determine white matter phenotypic data of MND at early stages of the disease for various types of MND. This will be useful as a biomarker to prognosticate the course and therefore choose patients for clinical trials and therapeutic interventions.

\section{Conclusion}

White matter abnormalities in this South Indian population of MND patients, seen in the form of reduced FA, was found in bilateral corticospinal tracts, bilateral anterior thalamic radiations, bilateral uncinate fasciculi, and right superior longitudinal fasciculus. Alteration in white matter integrity was present in not only motor tracts, but also several nonmotor association tracts indicating probable involvement of nonmotor neurons and tracts also in the pathological processes. DTI findings were more pronounced than what was visible with structural MRI. We did not find a significant correlation between FA values and disease severity (ALSFRS-R score) or disease duration.

\section{Funding}

None.

\section{Conflict of Interest}

None declared.

\section{Acknowledgment}

None.

\section{References}

1 Talbott EO, Malek AM, Lacomis D. The epidemiology of amyotrophic lateral sclerosis. Handb Clin Neurol 2016;138:225-238

2 Arthur KC, Calvo A, Price TR, Geiger JT, Chiò A, Traynor BJ. Projected increase in amyotrophic lateral sclerosis from 2015 to 2040. Nat Commun 2016;7:12408

3 Hong Y-H, Lee K-W, Sung J-J, Chang K-H, Song IC. Diffusion tensor MRI as a diagnostic tool of upper motor neuron involvement in amyotrophic lateral sclerosis. J Neurol Sci 2004;227(1):73-78

4 Woo JH, Wang S, Melhem ER, et al. Linear associations between clinically assessed upper motor neuron disease and diffusion 
tensor imaging metrics in amyotrophic lateral sclerosis. PLoS One 2014;9(8):e105753

5 Rajagopalan V, Pioro EP. Differential involvement of corticospinal tract (CST) fibers in UMN-predominant ALS patients with or without CST hyperintensity: a diffusion tensor tractography study. Neuroimage Clin 2017;14:574-579

6 Basaia S, Filippi M, Spinelli EG, Agosta F. White matter microstructure breakdown in the motor neuron disease spectrum: recent advances using diffusion magnetic resonance imaging. Front Neurol 2019;10:193

7 Cedarbaum JM, Stambler N, Malta E, et al. BDNF ALS Study Group (Phase III). The ALSFRS-R: a revised ALS functional rating scale that incorporates assessments of respiratory function. J Neurol Sci 1999;169(1-2):13-21

8 Prudlo J, Bißbort C, Glass A, et al. White matter pathology in ALS and lower motor neuron ALS variants: a diffusion tensor imaging study using tract-based spatial statistics. J Neurol 2012;259(9):1848-1859

9 Wang S, Melhem ER, Poptani H, Woo JH. Neuroimaging in amyotrophic lateral sclerosis. Neurotherapeutics 2011;8(1):63-71

10 Ahn S, Lee SK. Diffusion tensor imaging: exploring the motor networks and clinical applications. Korean J Radiol 2011;12(6):651-661

11 Prokscha T, Guo J, Hirsch S, et al. Diffusion tensor imaging in amyotrophic lateral sclerosis-increased sensitivity with optimized region-of-interest delineation. Clin Neuroradiol 2014;24(1):37-42

12 Sage CA, Van Hecke W, Peeters R, et al. Quantitative diffusion tensor imaging in amyotrophic lateral sclerosis: revisited. Hum Brain Mapp 2009;30(11):3657-3675

13 Metwalli NS, Benatar M, Nair G, Usher S, Hu X, Carew JD. Utility of axial and radial diffusivity from diffusion tensor MRI as markers of neurodegeneration in amyotrophic lateral sclerosis. Brain Res 2010;1348:156-164

14 Menke RAL, Abraham I, Thiel CS, et al. Fractional anisotropy in the posterior limb of the internal capsule and prognosis in amyotrophic lateral sclerosis. Arch Neurol 2012;69(11):1493-1499

15 Wang S, Melhem ER. Amyotrophic lateral sclerosis and primary lateral sclerosis: the role of diffusion tensor imaging and other advanced MR-based techniques as objective upper motor neuron markers. Ann N Y Acad Sci 2005;1064:61-77

16 Sarica A, Cerasa A, Vasta R, et al. Tractography in amyotrophic lateral sclerosis using a novel probabilistic tool: a study with tract-based reconstruction compared to voxel-based approach. J Neurosci Methods 2014;224:79-87

17 Jellison BJ, Field AS, Medow J, Lazar M, Salamat MS, Alexander AL. Diffusion tensor imaging of cerebral white matter: a pictorial review of physics, fiber tract anatomy, and tumor imaging patterns. AJNR Am J Neuroradiol 2004;25(3):356-369

18 Li J, Pan P, Song W, Huang R, Chen K, Shang H. A meta-analysis of diffusion tensor imaging studies in amyotrophic lateral sclerosis. Neurobiol Aging 2012;33(8):1833-1838

19 Trojsi F, Caiazzo G, Corbo D, et al. Microstructural changes across different clinical milestones of disease in amyotrophic lateral sclerosis. PLoS One 2015;10(3):e0119045

20 Kasper E, Schuster C, Machts J, et al. Microstructural white matter changes underlying cognitive and behavioural impairment in ALS-an in vivo study using DTI. PLoS One 2014;9(12):e114543

21 Agosta F, Pagani E, Petrolini M, et al. Assessment of white matter tract damage in patients with amyotrophic lateral sclerosis: a diffusion tensor MR imaging tractography study. AJNR Am J Neuroradiol 2010;31(8):1457-1461

22 Keil C, Prell T, Peschel T, Hartung V, Dengler R, Grosskreutz J. Longitudinal diffusion tensor imaging in amyotrophic lateral sclerosis. BMC Neurosci 2012;13:141

23 Barrio-Arranz G, de Luis-García R, Tristán-Vega A, Martín-Fernández M, Aja-Fernández S. Impact of MR acquisition parameters on DTI scalar indexes: a tractography based approach. PLoS One 2015;10(10):e0137905

24 Kalra S, Müller H-P, Ishaque A, et al. A prospective harmonized multicenter DTI study of cerebral white matter degeneration in ALS. Neurology 2020;95(8):e943-e952

25 Oouchi H, Yamada K, Sakai K, et al. Diffusion anisotropy measurement of brain white matter is affected by voxel size: underestimation occurs in areas with crossing fibers. AJNR Am J Neuroradiol 2007;28(6):1102-1106

26 Bede P, Bokde AL, Byrne S, et al. Multiparametric MRI study of ALS stratified for the C9orf72 genotype. Neurology 2013;81(4):361-369

27 Agosta F, Ferraro PM, Riva N, et al. Structural and functional brain signatures of C9orf72 in motor neuron disease. Neurobiol Aging 2017;57:206-219

28 Shamim U, Ambawat S, Singh J, et al. C9orf72 hexanucleotide repeat expansion in Indian patients with ALS: a common founder and its geographical predilection. Neurobiol Aging 2020;88:156.e1-156.e9 